\title{
Demonstration of Robertsonian Fusion by Silver Staining in a 13/14 Translocation in Man
}

\author{
S. M. Jalal, Mary L. Coleman and Walter A. Washahl \\ Department of Biology and the Department of Pathology, The Medical School, \\ of the University of North Dakota, Grand Forks, N. D. 58202, U.S.A.
}

Received April 22, 1980

Translocations, balanced or unbalanced, involving $D / D, D / G$ and $G / G$ are not uncommon in human populations. The incidence of $D / D$ translocations is estimated to be 1 in 1000 live births (Hamerton et al. 1972). Most of these translocations are between non-homolgous chromosomes of the D group with t (13q; 14q) being most frequent (Centerwall and Merrell 1975). The D/D and D/G type translocation Down's syndrome frequency is reported to be slightly less than three percent (Wright et al. 1967). Balanced translocation carriers such as the $D / D$, though phenotypically normal, have a high incidence of spontaneous abortions (Fernhoff et al. 1976).

Translocations can be a typical Robertsonian fusion where breaks occur in both non-homologous chromosomes and in the process of production of a biarmed chromosome a centromere is lost. Fusion between non-homologous chromosomes may occur with a break in only one chromosome, such as that reported for $7 / 22$ translocation (Larson et al. 1977). Translocations between non-homologous chromosomes may occur also without apparent breaks resulting in fusion between telomere to telomere (T-T), telomere and centromere (T-C) and centromere to centromere (C-C) in mammalian species that may result in a functional and a latent centromere in the dicentric chromosomes (Hsu et al. 1975). These are specialized type of Robertsonian fusions according to Hsu's classification.

The short arms of human acrocentric chromosomes of the $\mathrm{D}$ and $\mathrm{G}$ groups can be subdivided into three regions: the $\mathrm{C}$-positive centromeric region, the $\mathrm{C}$-negative secondary constriction and the $\mathrm{C}$-negative terminal satellite. The five pairs of acrocentric chromosomes with the secondary constriction regions are known to be involved in the production of nucleoli. Since the silver staining technique of Goodpasture and Bloom (1975) has now been greatly simplified (Lau et al. 1978), it is possible not only to identify the individual chromosomes involved in translocation but to determine if the nucleolar organizer regions (NOR) have been lost.

\section{Materials and methods}

A 26 year old mother who has a phenotypically normal $2 \frac{1}{2}$ year old girl had a second child that was stillborn with multiple congenital malformations. The abnormalities in the stillborn included, presence of extra digits, bilateral cleft palate and high interventricular septal defect. The parents were therefore advised to undergo cytogenetic examination. 
Lymphocyte culture from whole blood was used for standard karyotype analysis of both the parents. Slightly modified G banding procedure of Seabright (1972) as described elsewhere (Larson et al. 1977) was utilized for identification of chromosomes. The $\mathrm{C}$ banding was a slight modification (Jalal et al. 1974) of the procedure by Arrighi and Hsu (1971). The simplified silver staining technique of Lau et al. (1978) was used to stain the nucleolar organizer regions (Ag-NOR). The procedure involved a pre-treatment of air dried slides, in borate buffer $\left(0.1 \mathrm{M} \mathrm{Na} \mathrm{Na}_{4}\right.$, $0.005 \mathrm{M} \mathrm{Na}_{2} \mathrm{~B}_{4} \mathrm{O}_{7}$, pH 9.1) for 15-20 minutes followed by a brief rinse in distilled water and incubation in $50 \% \mathrm{AgNO}_{3}$ solution in a moist chamber for 18-22 hours at $55-60^{\circ} \mathrm{C}$.
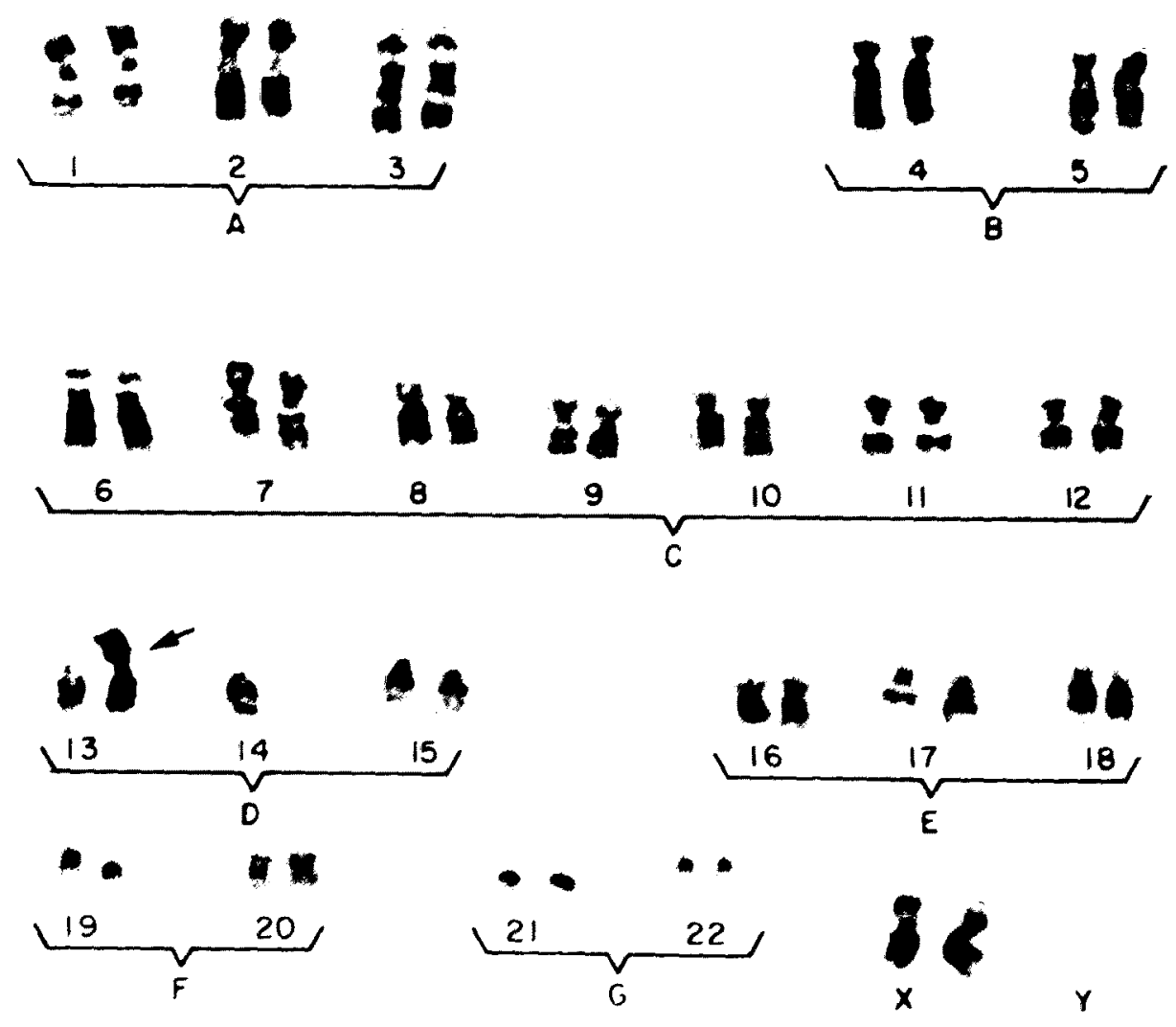

Fig. 1. G banded karyotype. Arrow indicates the $t(13 q ; 14 q)$ chromosome.

Results and discussion

Based on the analysis of 30-33 cells each the father had a normal diploid chrornosome complement, and the mother with a 45 chromosome count seemed to have a balanced translocation. Five G-banded karyotypes confirmed the observation that the mother had a $t(13 q ; 14 q)$ translocation (Fig. 1). The C-band of the translocated chromosome revealed a single centromere, though dicentric chromosomes 
are also known to occur in such translocations (Niebuhr 1972, Hsu et al. 1975).

Forty-five spreads were counted for nucleolar organizer regions (NOR) by silver staining. The number of NORs ranged from 3 to 8 with an average of 6.3 per cell (Table 1). The translocation product, $t(12 q ; 14 q)$ was devoid of any silver stain (Fig. 2) in all the cells examined. The break points must therefore be

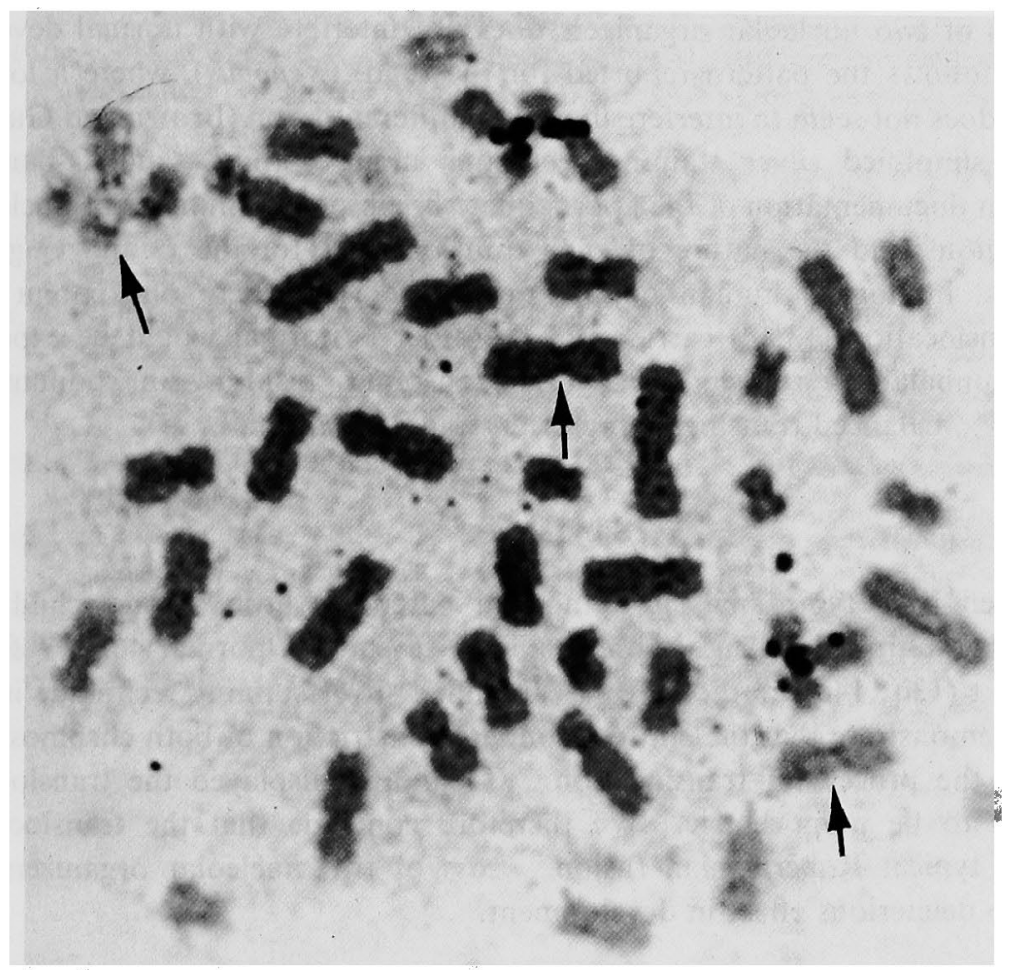

Fig. 2. Silver stained NORs from $4 \mathrm{G}$ and $3 \mathrm{D}$ group chromosomes. Arrow indicated the two No. 3 and an unstained translocated chromosome.

Table 1. Frequency of silver stained nucleolar organizers in a $13 / 14$ translocation

\begin{tabular}{cc}
\hline No. of cells & Ag-NOR/cell* \\
\hline 2 & 3 \\
3 & 4 \\
2 & 5 \\
14 & 6 \\
18 & 7 \\
6 & 8 \\
\hline
\end{tabular}

* Represents the total number of stained nucleolar organizers in the D and G group chromosomes.

in the centromeric regions so that the nucleolar organizers are lost from both chromosomes. Since the translocated chromosome seems to have a single centromere we conclude that this is a typical Robertsonian fusion. Another case of 13/14 
Robertsonian type translocation has been reported by Hurley and Pathak (1977). Gosden et al. (1979) report a loss of slightly over $60 \%$ of satellite DNA (Sat. III) in a 13/14 translocation where both nucleolar organizers were deleted. It is known that the ammonical silver staining technique (Goodpasture and Bloom 1975) corresponds closely to the sites of ribosomal cistron determined by "in situ" hybridization procedures (Pardue and Hsu 1975, Hsu et al. 1975). It is interesting that complete loss of two nucleolar organizers does not interfere with normal development. This follows the pattern reported for the toad, (Xenopus), where a loss of $50 \% \mathrm{r}$ DNA does not seem to interfere in normal differentiation (Brown and Gurdon 1964). The simplified silver staining technique along with $C$ - and $G$-banding should help in documentation of the approximate break points, number of nucleolar organizer regions and the nature of such translocations for the D and $G$ group chromosomes, in normal human populations. The risk where one parent is a balanced translocation (13/14) carrier and the other is normal would be expected to be $50 \%$ unbalanced translocations or monosomics resulting in spontaneous abortions, $25 \%$ balanced translocation carriers and $25 \%$ normal.

\section{Summary}

$G$ banded karyotypic analysis of normal parents, whose second child was stillborn with multiple congenital abnormalities, revealed a normal 46, XY father and $45, X X, t(13 q ; 14 q)$ mother. By a simplified silver staining technique it was possible to demonstrate that the entire secondary constriction of both chromosomes were lost in the process of translocation. $\mathrm{C}$ banding displayed the translocated chromosome to be monocentric. We therefore conclude that the translocation represents a typical Robertsonian fusion. Loss of two nucleolar organizers has seemingly no deleterious effect in development.

\section{Acknowledgements}

The authors wish to acknowledge the assistance of Dr. C. J. Dillenburg of the Pathology Department who performed the autopsy on the stillborn and Professor Jean Saumur for her help in preparation of the manuscript and encouragement for local cytogenetic research.

\section{References}

Arrighi, F. E. and Hsu, T. C. 1971. Localization of heterochromatin in human chromosomes. Cytogenetics 10: 81-86.

Brown, D. D. and Gurdon, J. B. 1964. Absence of ribosomal RNA synthesis in the anucleate mutant of Xenopus laevis. Proc. Nat. Acad. Sci. (U.S.A.) 51 : 131-146.

Centerwall, W. R. and Merrell, P. R. 1975. Familial D/D translocation t (13q: 14q) eight members in four generations. Clin. Genet. 7: 91-97.

Fernhoff, P, M., Singh, D. M., Hanson, J., Strucler, S., Dumong, C. R., and Chen, A.T.L. 1976. Association of D/D translocation with fetal wastage and aneupolidy. J. Med. Genet. 13: 389-393.

Goodpasture, C. and Bloom, S. E. 1975. Visualization of nucleolar organizer regions in mam- 
malian chromosomes using silver staining. Chromosoma (Berl.) 53: 37-50.

Gosden, J. R., Gosden, C. M., Lawrie, S. S. and Buckton, K. E. 1979. Satellite DNA loss and nucleolar organizer activity in an individual with a de novo chromosome 13,14 translocation. Clin. Genet. 15: 518-529.

Hamerton, J. L., Abbot, J., Ray M., Williamson, C. L. and Ducasse, G. C. 1972. Chromosome studies in a neonatal population. Canad. Med. Ass. J. 106: 776-779.

Hsu, T. C., Spirito, S. E. and Pardue, M. L. 1975. Distribution of $18+28$ S ribosomal genes in mammalian genomes. Chromosoma (Berl.) 53: 25-36.

-, Pathak, S. and Chen, T. R., 1975. The possibility of latent centromeres and a proposed nomenclature system for total chromosome and whole arm translocations. Cytogenet. Cell genet. 15 : 41-49.

Hurley, J. E., and Pathak, S. 1977. Elimination of necleolus organizers in a case of 13/14 Robertsonian translocation. Human Genet. 35: 169-173.

Jalal, S. M., Pfeiffer, R. A., Pathak, S. and Hsu, T. C. 1974 . Subdivision of human Y chromosome. Humangenetik 24: 59-65.

Lau, Y. F., Pfeiffer, R. A., Arrighi, F. E. and Hsu, T. C. 1978. Combination of silver and fluorescent staining for metaphase chromosomes. Amer. J. Hum. Genet. 30: 76-79.

Larson, L. M., Wasdahl, W. A. and Jalal, S. M. 1977. Partial trisomy 7p associated with familial 7p; 22 q translocation. J. Med. Genet. 14: 258-261.

Niebuhr, E. 1972. Dicentric and monocentric Robertsonian translocations in man. Hum. Genet. 16: $217-226$.

Pardue, M. L., and T. C., Hsu, 1975. Locations of $18 \mathrm{~S}$ and $28 \mathrm{~S}$ ribosomal genes on the chromosomes of Indian Muntjac. J. Cell. Biol, 64: 251-254.

Seabright, M. 1971. A rapid banding technique for human chromosomes. Lancet 2: 971-972.

Wright, S. W., Day, R. W., Muller, H. and Weinhouse, R. 1967. The frequency of trisomy and translocation in Down's syndrome. J. Pediat. 70: 420-424. 